\title{
TIPE1 impairs ovarian tumor growth by promoting caspase-dependent apoptosis
}

\author{
TINGTING LI ${ }^{1,2}$, LEI JIA ${ }^{2,3}$, YU DENG ${ }^{4}$, BO WANG ${ }^{2}$ and SONG QUAN ${ }^{1}$ \\ ${ }^{1}$ Department of Gynecology and Obstetrics, Center for Reproductive Medicine, Nanfang Hospital, Southern \\ Medical University, Guangzhou, Guangdong 510515; ${ }^{2}$ Reproductive Medicine Research Center, Sixth Affiliated \\ Hospital of Sun Yat-Sen University, Guangzhou, Guangdong 510655; ${ }^{3}$ Laboratory Medicine Center, Nanfang \\ Hospital, Southern Medical University, Guangzhou, Guangdong 510515; ${ }^{4}$ Assisted Reproductive Medical \\ Center, Guangzhou Women and Children's Hospital, Guangzhou, Guangdong 510623, P.R. China
}

Received January 18, 2020; Accepted September 14, 2020

DOI: $10.3892 / \mathrm{ol} .2020 .12227$

\begin{abstract}
Tumor necrosis factor- $\alpha$-induced protein 8 -like 1 (TIPE1) functions as a tumor suppressor in several types of cancer, including lung and breast cancer. The present study aimed to determine the level of expression and the function of TIPE1 in ovarian cancer. TIPE1 expression was determined in tissue microarrays and ovarian cancer cells, and these data were analyzed to assess the association between TIPE1 expression and prognosis in patients with ovarian cancer. The potential antitumor effects of TIPE1 were investigated in vitro and in a xenograft mouse model. Furthermore, the underlying molecular mechanism by which TIPE1 regulates ovarian cancer growth was determined via flow cytometric analysis, western blotting and rescue experiments. The results of the present study indicated that TIPE1 levels were markedly decreased in ovarian cancer tissues, and its level of expression was associated with a favorable prognosis of patients with ovarian cancer. In addition, ectopic TIPE1 expression significantly impaired A2780 and SKOV3 cell proliferation and colony formation in vitro, which was accompanied by efficient inhibition of xenograft tumor growth in mice. Investigations into the underlying molecular mechanism demonstrated that TIPE1 induced ovarian cancer cell apoptosis by promoting caspase protein expression. Inhibition of caspase-dependent apoptosis by z-VAD blocked TIPE1-mediated inhibition of the proliferation and induction of apoptosis in ovarian cancer cells. Collectively, the results of the present study suggest that TIPE1 may be a potential prognostic predictor and therapeutic target for patients with ovarian cancer.
\end{abstract}

Correspondence to: Dr Song Quan, Department of Gynecology and Obstetrics, Center for Reproductive Medicine, Nanfang Hospital, Southern Medical University, 1838 Guangzhou Avenue North, Guangzhou, Guangdong 510515, P.R. China

E-mail: quansongnfsu@163.com

Key words: ovarian cancer, TIPE1, apoptosis, caspase, prognosis

\section{Introduction}

Ovarian cancer is the sixth most frequently diagnosed cancer among women worldwide (11.8 cases per 100,000 women in 2014), the second most common gynecologic malignancy in female patients, and the most fatal tumor of the female reproductive system $(1,2)$. Among of ovarian tumors, $~ 90 \%$ are epithelial ovarian serous cancers that occur primarily in postmenopausal women $(3,4)$. Despite the high response rates in several patients receiving initial chemotherapy, the majority of patients with advanced ovarian cancer ultimately develop recurrent disease that is resistant to chemotherapy $(5,6)$. The identification of biomarkers has contributed to the management of ovarian cancer by enabling the monitoring of patients' response to treatment and recurrence, distinction of benign and malignant pelvic masses, and detection of the disease at an earlier stage $(7,8)$. Thus, the identification of novel biomarkers would benefit the prediction of patient prognosis and the development of novel therapeutic strategies.

The tumor necrosis factor- $\alpha$-induced protein 8 -like (TIPE/TNFAIP8) family is a recently identified family of proteins that participate in the regulation of immunity and tumorigenesis (9). The family comprises four members: TIPE, TIPE1, TIPE2 and TIPE3 (9). TIPE2 has been demonstrated to be a negative regulator of inflammation and cellular immunity $(10,11)$, whereas TIPE expression is downregulated in several malignances, such as lung cancer, and is associated with a favorable patient prognosis $(12,13)$. TIPE1 is considered a cell death inducer in both normal and cancer cells $(12,13)$. Downregulated TIPE1 expression has been observed in hepatocellular carcinoma (14), lung cancer (15) and gastric cancer (16). Ectopic TIPE1 expression promotes apoptosis and inhibits cell invasion and migration in several types of cancer (14-16); however, in cervical cancer, TIPE1 facilitates cell proliferation and suppresses the susceptibility of cells to cisplatin chemotherapy (17). Furthermore, ectopic TIPE1 expression significantly impairs osteosarcoma tumor growth in vivo by inhibiting macrophage infiltration (18). However, the role of TIPE1 in ovarian serous cancer remains unclear.

The present study aimed to determine the level of expression and function of TIPE1 in ovarian cancer, using in vitro 
and xenograft models. In addition, the underlying molecular mechanism by which TIPE1 regulates ovarian cancer growth was investigated via flow cytometric analysis, western blotting and rescue experiments. The results of the present study expand the understanding of TIPE1 and provide a potential prognostic predictor and therapeutic target for ovarian cancer.

\section{Materials and methods}

Cell culture and reagents. Ovarian cancer cells (A2780, PA-1 and SKOV3) were purchased from the American Type Culture Collection, while the fallopian tube secretory epithelial cells (FTSECs) were purchased from Shanghai $\mathrm{Yu}$ Bo Biotech Co., Ltd. Cells were cultured in DMEM or RPMI-1640 medium supplemented with $10 \%$ fetal calf serum (all from Invitrogen; Thermo Fisher Scientific, Inc.) at $37^{\circ} \mathrm{C}$ with $5 \% \mathrm{CO}_{2}$. The lentivirus-based TIPE1 expression system (based on the LV6-EF1 $\alpha$-Puro vector) and an empty vector control system were purchased from Shanghai GenePharma Co., Ltd. and were used to infect the ovarian cancer cells. Briefly, $1 \times 10^{5}$ A2780 and SKOV3 cells were seeded into each well of a 6-well plate and incubated at $37^{\circ} \mathrm{C}$ with $5 \% \mathrm{CO}_{2}$. At $24 \mathrm{~h}$, $2 \times 10^{6}$ lentivirus $(\mathrm{MOI}=20)$ in $20 \mu 1$ was added to infect the cells at $37^{\circ} \mathrm{C}$ with $5 \% \mathrm{CO}_{2}$, and $24 \mathrm{~h}$ later the culture medium was removed. The stably infected cells were selected by treatment with $2 \mu \mathrm{g} / \mathrm{ml}$ puromycin (Selleck Chemicals) for $48 \mathrm{~h}$ at $37^{\circ} \mathrm{C}$ with $5 \% \mathrm{CO}_{2}$. The caspase-specific inhibitor Z-VAD was purchased from Selleck Chemicals and dissolved in DMSO for ovarian cancer cell treatment $(20 \mu \mathrm{M})$ for $24 \mathrm{~h}$ at $37^{\circ} \mathrm{C}$ with $5 \% \mathrm{CO}_{2}$ after selecting the stably infected cells.

Tissue microarray. A microarray containing 90 human malignant ovarian cancer tissues and 29 adjacent normal tissues ( $>5 \mathrm{~cm}$ away from the tumor) were purchased from Shanghai Outdo Biotech Co., Ltd. IHC staining was performed as previously described (19). Briefly, the tissue microarray was subjected to dewaxing and hydration. Following antigen retrieval, the slides were incubated with primary antibodies against TIPE1 (cat. no. 201986; 1:200 dilution; Abcam) overnight at $4^{\circ} \mathrm{C}$. Following incubation with the specific secondary antibodies (cat. nos. SP9001 and SP9002; OriGene Technologies, Inc.) at $37^{\circ} \mathrm{C}$ for $1 \mathrm{~h}$, the bound antibodies were visualized using 3,3'-diaminobenzidine (Fuzhou Maixin Biotech Co., Ltd.). The nuclei were counterstained with hematoxylin at room temperature for $10 \mathrm{~min}$. The TIPE1 staining in the tumor sections was scored by counting the number of positive cells. TIPE1 staining was scored based on the percentage of TIPE1-positive cells as follows: 0 points, $<5 \% ; 1$ point, $>5 \%$ and $<15 \%$; 2 points, $>15 \%$ and $<25 \%$; 3 points, $>25 \%$ and $<35 \%$; 4 points, $>35 \%$. The tumors with a score of 0 or 1 were classified as TIPE1 low-expression tumors, while those with a score of 2-4 were classified as TIPE1 high-expression tumors. The analysis of the association between TIPE1 expression and patient prognosis was performed as previously described (20).

Bioinformatics analyses. The expression raw data of TIPE1 in ovarian tumors $(n=98)$ and adjacent normal tissues $(n=20)$ was downloaded from The Cancer Genome Atlas (TCGA) database (https://www.cancer.gov/about-nci/organization/ccg/research/structural-genomics/tcga). Relative TIPE1 expression for each sample was calculated after normalizing it to one normal sample. Wilcoxon signed-rank test was used to analyze the data.

Cell viability assay. Cell Counting Kit-8 (CCK-8; Beyotime Institute of Biotechnology) assay was performed to measure cell viability, according to the manufacturer's instructions. Briefly, 1,000 cells/well were seeded into 96-well plates, and cell viability was measured using CCK-8 at $0,24,48$ and $72 \mathrm{~h}$ by measuring absorbance at $450 \mathrm{~nm}$ using a microplate reader. A total of four independent experiments were performed for each group.

Western blotting. Ovarian cancer cells and tumor tissues were collected and lysed using RIPA lysis buffer (Beyotime Institute of Biotechnology) supplemented with $1 \%$ protease inhibitor cocktail (Sigma-Aldrich; Merck KGaA). Following centrifugation at $12,000 \mathrm{x} \mathrm{g}$ for $15 \mathrm{~min}$ at $4^{\circ} \mathrm{C}$, the supernatants were collected for protein concentration determination via the Bradford assay (Beyotime Institute of Biotechnology). A total of $20 \mu \mathrm{g}$ of protein was loaded for electrophoretic separation on $10 \%$ SDS/polyacrylamide gels and transferred onto PVDF membranes (MilliporeSigma). Membranes were blocked with 5\% milk in TBS/Tween-20 (0.1\%) buffer at room temperature for $2 \mathrm{~h}$, and subsequently probed with primary antibodies against: TIPE1 (cat. no. 201986; 1:600 dilution; Abcam), caspase-3 (cat. no. 9662; 1:400 dilution), caspase-8 (cat. no. 4927, 1:500 dilution), caspase-9 (cat. no. 9502; 1:400 dilution), cleaved poly(ADP-ribose) polymerase (PARP; cat. no. 9548; 1:400 dilution), total PARP (cat. no. 5625; 1:1,000 dilution) and GAPDH (cat. no. 5174; 1:2,000 dilution) (all from Cell Signaling Technology, Inc.). Following the primary incubation, membranes were incubated with horseradish peroxidase-conjugated anti-rabbit immunoglobulin $\mathrm{G}(\mathrm{IgG}) /$ anti-mouse $\mathrm{IgG}$ secondary antibody (cat. no. ZDR-5306 and ZDR-5307; 1:10,000 dilution; OriGene Technologies, Inc.) at room temperature for $2 \mathrm{~h}$, and an ECL kit (MilliporeSigma) was used to detect the expression of the target proteins.

Colony formation assay. For the colony formation assay, 2,000 ovarian cancer cells were seeded into 6-well plates containing $2 \mathrm{ml}$ DMEM containing 10\% FBS. Cells were subsequently cultured in a humidified atmosphere of $5 \% \mathrm{CO}_{2}$ at $37^{\circ} \mathrm{C}$ for 10-14 days. The plated cells were then fixed with $4 \%$ paraformaldehyde at room temperature $\left(22-25^{\circ} \mathrm{C}\right)$ for $15 \mathrm{~min}$, and stained with $0.2 \%$ crystal violet (Beyotime Institute of Biotechnology) for $15 \mathrm{~min}$ at room temperature. After washing with distilled water, the colonies in each well were counted.

$R N A$ isolation and reverse transcription-quantitative $(R T-q)$ $P C R$. Total RNA was extracted using TRIzol ${ }^{\circledR}$ reagent (Invitrogen; Thermo Fisher Scientific, Inc.), according to the manufacturer's instructions. The concentration of RNA was determined by NanoDrop 2000 (Thermo Fisher Scientific, Inc.) and a total of $1 \mu \mathrm{g}$ RNA was used for RT using a Reverse Transcription kit (cat. no. RR045A; Takara Bio, Inc.) according to the manufacturer's instructions. For qPCR amplification, the reaction was performed in a $20 \mu \mathrm{l}$ reaction volume containing $10 \mu \mathrm{l}$ SYBR-Green 
PCR Master mix (cat. no. RR087A; Takara Bio, Inc.), using a real-time PCR system (Roche Bioscience). The thermocycling conditions were as follows: 1 cycle at $95^{\circ} \mathrm{C}$ for $30 \mathrm{sec}$, followed by 42 cycles at $95^{\circ} \mathrm{C}$ for $5 \mathrm{sec}$ and $58^{\circ} \mathrm{C}$ for $30 \mathrm{sec}$. GAPDH was used as a loading control to normalize TIPE1 expression. qPCR was subsequently performed, as previously described (21). The following primer sequences were used for qPCR: TIPE1 forward, 5'-CAGTGACCTGCTAGATGAG-3', and reverse, 5'-CAAGGTGCTGAGTGAAGT-3'; and GAPDH forward, 5'-GGTGAAGGTCGGAGTCAACG-3', and reverse, 5'-CAAAGTTGTCATGGATGACC-3'. The results were analyzed as previously described (22).

Flow cytometric analysis of apoptosis and cell cycle. Ovarian cancer cells were seeded into a 6-well plate at a density of $5 \times 10^{5}$ cells/well, and subsequently incubated with $20 \mathrm{nM}$ z-VAD for $24 \mathrm{~h}$ at $37^{\circ} \mathrm{C}$. Cells were collected by centrifugation at $1,500 \mathrm{x} \mathrm{g}$ at room temperature for $3 \mathrm{~min}$, washed with ice-cold phosphate buffered saline (PBS) three times, and fixed with ice-cold $70 \%$ ethanol for $15 \mathrm{~min}$ at $4^{\circ} \mathrm{C}$. For apoptosis analysis, the apoptotic cells were stained with Annexin V-fluorescein isothiocyanate and propidium iodide (PI; Nanjing KeyGen Biotech Co., Ltd.), according to the manufacturer's instructions. For cell cycle analysis, the cells were stained with propidium iodide (cat. no. C1052; Beyotime Institute of Biotechnology) according to the manufacturer's instructions. Apoptotic cells and the cell cycle were subsequently analyzed using a FACSCanto II flow cytometer (BD Biosciences). The results were analyzed by FlowJo software (version 10.0; FlowJo, LLC).

Animal study. All animal experiments were performed in compliance with the provisions of the regulation for the Care and Use of Laboratory Animals of Southern Medical University (Guangzhou, China). Balb/c female nude mice (age, 5-6 weeks; weight, 15-16 g; 6 mice/group) were purchased from Beijing HFK Bioscience Co., Ltd., and maintained under specific pathogen-free conditions, with free access to water and sterile food at $22-25^{\circ} \mathrm{C}$ and 12 -h light/dark cycle. To establish xenograft mouse models, the ovarian cancer cells were collected and washed three times with sterile PBS. After counting the cell number, cells were suspended in sterile PBS $\left(5 \times 10^{7} \mathrm{cells} / \mathrm{ml}\right)$, and $100 \mu \mathrm{l}$ was subcutaneously inoculated into the ventral of the mice. The tumor width and length were measured from day 10 to day 25 post-injection using a vernier caliper. The tumor volumes were calculated as follows: Tumor volume $=$ length $\mathrm{x}$ width ${ }^{2} \times 0.52$. No multiple tumors were presented. At day 25 post-injection, the mice were anesthetized by intraperitoneal injection with $60 \mu 110 \%$ chloral hydrate $(300 \mathrm{mg} / \mathrm{kg}$; Beyotime Institute of Biotechnology) and sacrificed by cervical dislocation. After confirming that the heartbeat of the mice had stopped, the tumors were collected for further analysis.

Immunohistochemical (IHC) staining and terminal-deoxynucleoitidyl transferase mediated nick end labeling (TUNEL) assay. IHC staining was performed, as previously described (19). Briefly, ovarian tumor tissues were fixed with $4 \%$ paraformaldehyde at room temperature for $48 \mathrm{~h}$ and embedded in paraffin. The $4-\mu \mathrm{m}$ slides were sectioned and subjected to dewaxing and hydration. Following antigen retrieval, the slides were incubated with primary antibodies against TIPE1 (cat. no. 201986; 1:200 dilution; Abcam) and caspase-3 (cat. no. 9662; 1:100 dilution; Cell Signaling Technology, Inc.) overnight at $4^{\circ} \mathrm{C}$. Following incubation with the specific secondary antibodies (cat. nos. SP9001 and SP9002; OriGene Technologies, Inc.) at $37^{\circ} \mathrm{C}$ for $1 \mathrm{~h}$, the bound antibodies were visualized using 3,3'-diaminobenzidine (Fuzhou Maixin Biotech Co., Ltd.). The nuclei were counterstained with hematoxylin at room temperature for $10 \mathrm{~min}$. TUNEL assay was performed according to the manufacturer's instructions (cat. no. QIA39; MilliporeSigma). Briefly, following dewaxing and hydration, the slides were incubated with $2 \mathrm{mg} / \mathrm{ml}$ Proteinase $\mathrm{K}$ (1:100 in $10 \mathrm{mM}$ Tris, $\mathrm{pH} 8$ ) at $37^{\circ} \mathrm{C}$ for $30 \mathrm{~min}$. After washing with TBS three times, the slides were incubated with $1 \mathrm{X}$ TdT Equilibration Buffer at room temperature for $15 \mathrm{~min}$ and with $1 \mathrm{X}$ TdT Labeling Reaction Mixture at $37^{\circ} \mathrm{C}$ for $1.5 \mathrm{~h}$. After washing with TBS three times, the slides were covered with mounting medium containing a cell nuclear stain. TUNEL-positive cells were observed in six randomly selected fields under a BX51 fluorescence microscope The number of TUNEL-positive cells per frame were counted by two experimenters.

5-Bromo-2-deoxyuridine staining (BrdU) staining. A2780 cells $\left(1 \times 10^{4}\right)$ were seeded into each well of a 6-well plate. At $24 \mathrm{~h}$, the cells were treated with $10 \mu \mathrm{M}$ BrdU (Beyotime Institute of Biotechnology) at $37^{\circ} \mathrm{C}$ for $1 \mathrm{~h}$, fixed with $2 \%$ formaldehyde for $10 \mathrm{~min}$ at room temperature, washed with $1 \mathrm{X}$ PBS, permeabilized in $0.2 \%$ Triton X-100 for $10 \mathrm{~min}$ at room temperature, and washed with $1 \mathrm{X}$ PBS. The cells were incubated with a phycoerythrin-conjugated-BrdU antibody (cat. no. 50230; 1:500 dilution; Cell Signaling Technology, Inc.) at $37^{\circ} \mathrm{C}$ for $2 \mathrm{~h}$. The cell nuclei were stained with DAPI (Beyotime Institute of Biotechnology). The positive cells were observed in four randomly selected fields under a BX51 fluorescence microscope.

Statistical analysis. Statistical analysis was performed using GraphPad Prism 5 (GraphPad Software, Inc.) and data are presented as the mean \pm standard deviation differences between two groups were evaluated using Student's t-test, and differences among multiple groups were evaluated using analysis of variance, followed by Tukey's multiple comparison test. The paired t-test was used for analyzing the IHC score in paired samples, and Wilcoxon signed-rank test was performed for TCGA data analysis. Kaplan-Meier followed by log-rank test was performed to analyze the rate of disease-free survival and overall survival. $\mathrm{P}<0.05$ was considered to indicate a statistically significant difference.

\section{Results}

Downregulation of TIPE1 predicts the progression of ovarian cancer. To investigate TIPE1 expression in ovarian cancer, IHC staining was performed with a tissue microarray containing normal and malignant ovarian tissues. TIPE1 expression was observed in normal ovarian tissues, whereas few TIPE1-positive cells were observed in malignant tissues (Fig. 1A). Further statistical analysis demonstrated a significant decrease in 
A

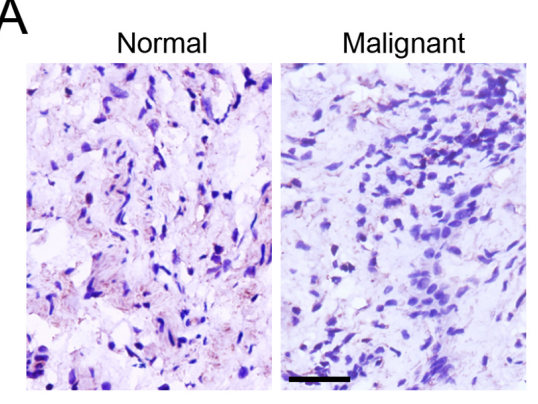

$\mathrm{D}$
B

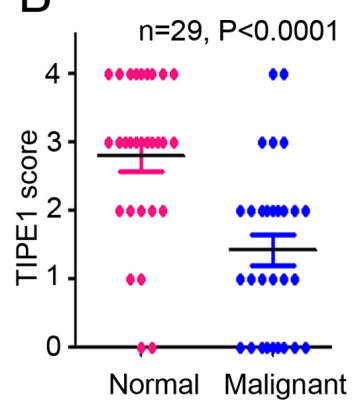

C

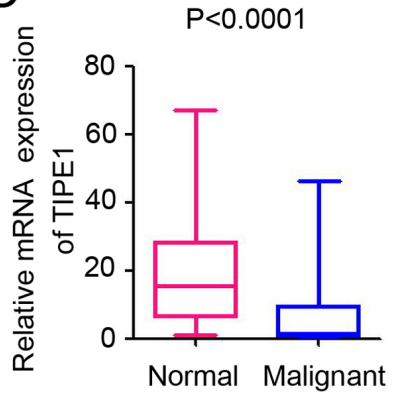

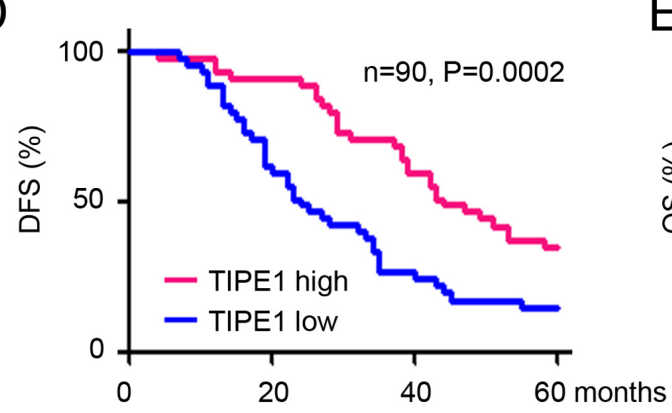

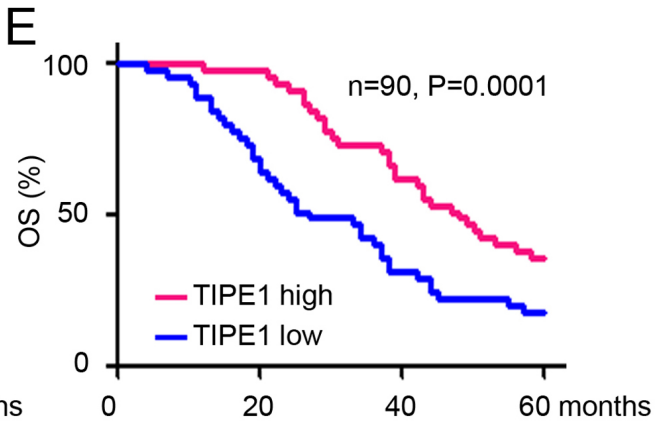

Figure 1. Downregulation of TIPE1 predicts progression of patients with ovarian cancer. (A) IHC staining of TIPE1 expression in a tissue microarray containing 90 ovarian cancer tissues and 29 forward normal tissues. Scale bar, $100 \mu \mathrm{m}$. (B) IHC staining score of TIPE1 in 29 pairs of normal and malignant ovarian tissues. (C) Relative TIPE1 expression in normal and ovarian serous malignant tissues from The Cancer Genome Atlas database. (D) Log-rank test analysis of the DFS of patients with ovarian cancer, which were divided according to TIPE1 expression in malignant tissues. (E) Log-rank test analysis of the OS of patients with ovarian cancer, which were divided according to TIPE1 expression in malignant tissues. TIPE1, tumor necrosis factor- $\alpha$-induced protein 8-like 1; IHC, immunohistochemical; DFS, disease-free survival; OS, overall survival.

TIPE1 expression in malignant ovarian tissues compared with normal tissues, based on the IHC scores (Fig. 1B). A similar downregulation of TIPE1 was also observed in the ovarian serous tumors based on TCGA database (Fig. 1C). To better understand the potential role of TIPE1 in predicting the prognosis of patients with ovarian cancer, patients were divided into high and low TIPE1 groups, respectively, according to IHC scores of TIPE1 expression. As presented in Fig. 1D, patients with a higher level of TIPE1 expression had a higher rate of disease-free survival than patients with lower levels of TIPE1 expression. Furthermore, lower TIPE1 expression levels were associated with poorer overall survival in patients with ovarian cancer (Fig. 1E). Collectively, these results demonstrated that TIPE1 expression was decreased in ovarian cancer tissues, and its expression levels were associated with a favorable prognosis of patients with ovarian cancer.

TIPE1 inhibits ovarian cancer cell proliferation in vitro. To select the best cells for determining the functional role of TIPE1 in ovarian cancer, TIPE1 expression was analyzed in normal human FTSECs and ovarian cancer cells (A2780, PA-1 and SKOV3) via western blotting and RT-qPCR analyses. TIPE1 protein and mRNA expression levels were downregulated in ovarian cancer cells (Fig. 2A and B). Subsequently, A2780 and SKOV3 cells, two ovarian cancer cell lines with low TIPE1 expression, were infected with a lentivirus based TIPE1 expression system or a control system. Following selection with puromycin, the stably infected cells were designated as A2780-Ctrl, A2780-TIPE1, SKOV3-Ctrl and SKOV3-TIPE1, and expression was confirmed via western blotting, which exhibited overexpression of TIPE1 in A2780-TIPE1 and
SKOV3-TIPE1 cells compared with the controls (Fig. 2C). In order to determine the cell viability of A2780 and SKOV3 cells, CCK-8 assay was performed. As presented in Fig. 2D and E, ectopic TIPE1 expression markedly inhibited the proliferation of A2780 and SKOV3 cells. Colony formation assays also confirmed the inhibitory role of TIPE1 on A2780 and SKOV3 cell proliferation in vitro (Fig. $2 \mathrm{~F}$ and $\mathrm{G}$ ). Taken together, these results provide evidence of the inhibitory role of TIPE1 in ovarian cancer cell viability.

TIPE1 promotes caspase-dependent apoptosis in ovarian cancer. To investigate the potential anticancer mechanisms of TIPE1 control, dual staining using PI and Annexin V was performed in ovarian cancer cells. The results demonstrated that TIPE1 effectively promoted apoptosis of both A2780 and SKOV3 cells (Fig. 3A and B; A2780-Ctrl, $3.2 \pm 1.0 \%$ vs. A $2780-\mathrm{TIPE} 1,10.7 \pm 1.4 \%$; SKOV3-Ctrl, $4.4 \pm 0.4 \%$ vs. SKOV3-TIPE1, $9.8 \pm 1.5 \%$ ). In addition, flow cytometric analysis indicated that TIPE1 had no significant effect on the distribution of ovarian cancer cell cycle (Fig. S1A). BrdU staining also indicated that there was no significant difference on the proliferation between A2780-Ctrl and A2780-TIPE1 cells (Fig. S1B). Western blot analysis demonstrated that TIPE1 promoted the expression of caspase-8, -9, -3, cleaved caspase-3, cleaved caspase- 9 and cleaved PARP in both A2780 and SKOV3 cells (Fig. 3C), and had no significant effects on total PARP expression (Fig. 3C). Following inhibition of caspase-dependent apoptosis using the specific inhibitor z-VAD, TIPE1-mediated inhibition of proliferation (Fig. 3D) and induction of apoptosis (Fig. 3E) in ovarian cancer cells was attenuated. Collectively, these 

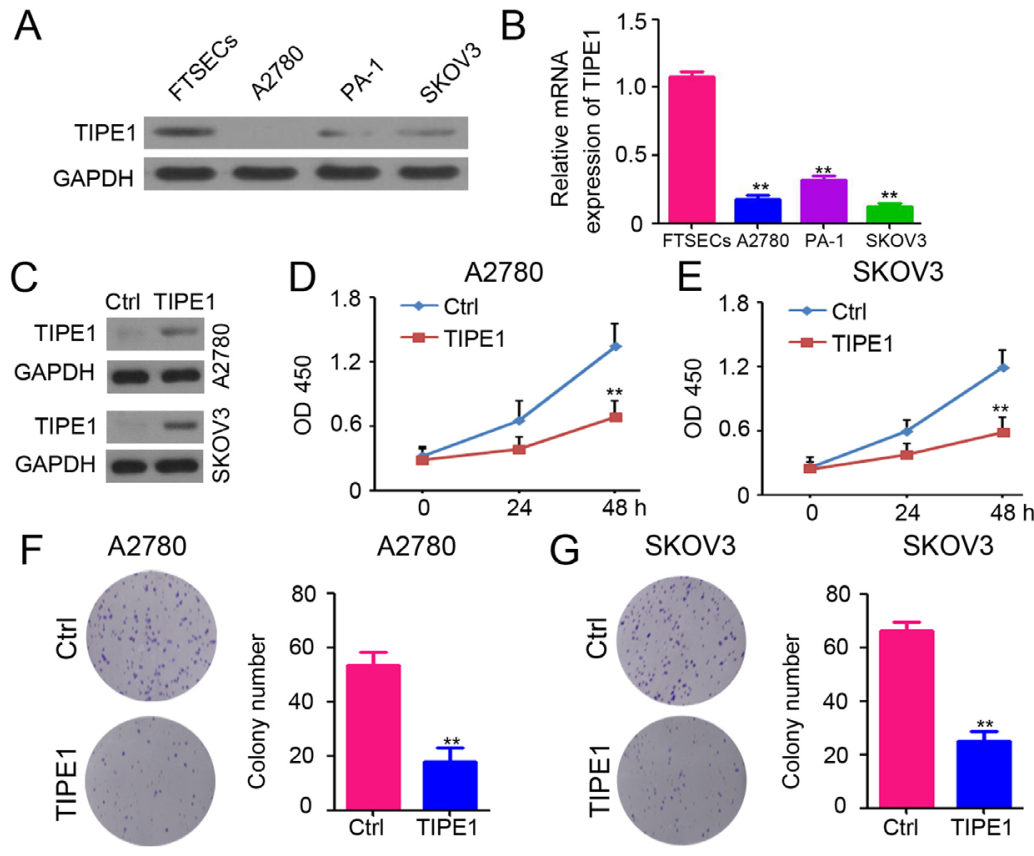

Figure 2. TIPE1 inhibits ovarian cancer cell proliferation in vitro. (A) Western blot analysis of TIPE1 expression levels in FTSECs and ovarian cancer cells (A2780, PA-1 and SKOV3). GAPDH was used as a loading control. (B) Relative mRNA expression of TIPE1 was determined via reverse transcription-quantitative PCR (n=3/group). (C) Western blotting analysis of TIPE1 expression in A2780 and SKOV3 cells that were stably infected with TIPE1 and Ctrl plasmids. GAPDH was used as a loading control. (D and E) Detection of cell viability by CCK-8 assay in A2780 and SKOV3 cells that were stably infected with TIPE1 and Ctrl plasmids ( $\mathrm{n}=4$ /group) at 0,24 and $48 \mathrm{~h}$ post cell plating. ( $\mathrm{F}$ and $\mathrm{G}$ ) Detection of colony formation in A2780 and SKOV3 cells that were stably infected with TIPE1 and Ctrl plasmids. The colonies in each well were counted and analyzed ( $\mathrm{n}=4 /$ group). ${ }^{* *} \mathrm{P}<0.01$ vs. FTSECs and Ctrl. TIPE1, tumor necrosis factor- $\alpha$-induced protein 8 -like 1; FTSECs, fallopian tube secretory epithelial cells; Ctrl, control; OD, optical density.

A

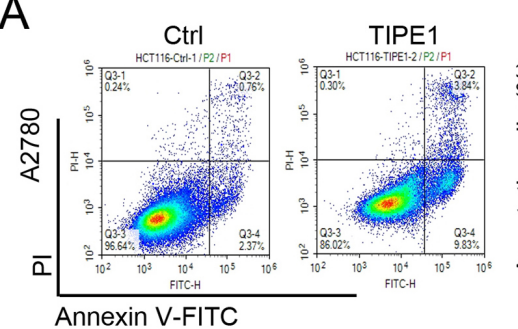

B

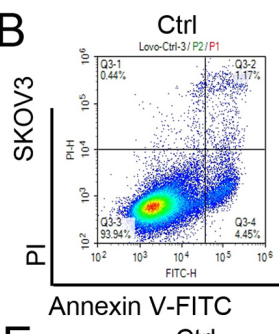

$\mathrm{E}$

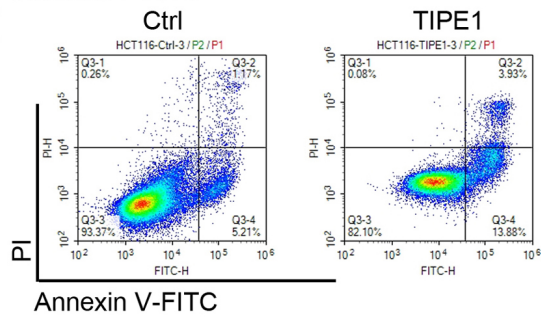

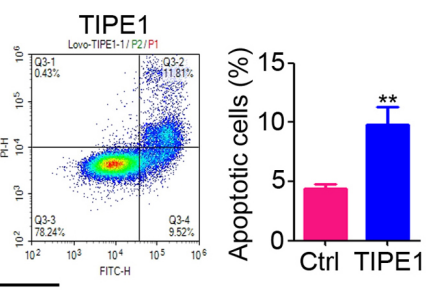

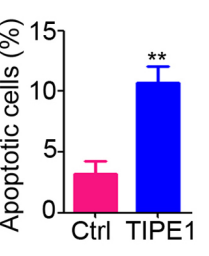

C

cleaved PARP

total PARP

Caspase 8

Caspase 9

CC9

Caspase 3

$\mathrm{CC} 3$

GAPDH

$\mathrm{D}$

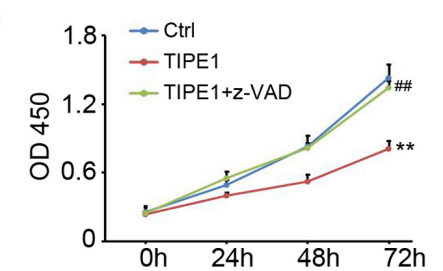

TIPE1+Z-VAD
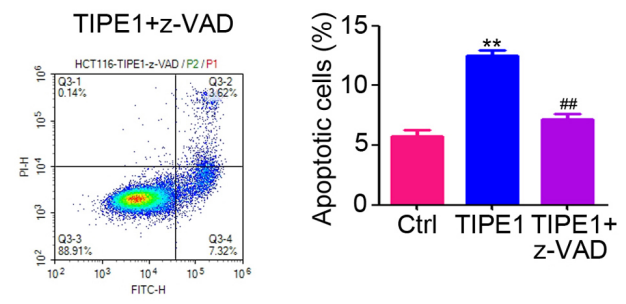

Figure 3. TIPE1 promotes caspase-dependent apoptosis in ovarian cancer. (A and B) Determination of apoptotic cells by PI and Annexin V staining in A2780-Ctrl, A2780-TIPE1, SKOV3-Ctrl and SKOV3-TIPE1 cells, followed by flow cytometry analysis. The percentage of apoptotic cells was analyzed $(\mathrm{n}=3)$. (C) Western blot analysis of caspase-8, caspase-9, CC9, caspase-3, CC3, total PARP and cleaved PARP expression in A2780-Ctrl, A2780-TIPE1, SKOV3-Ctrl and SKOV3-TIPE1 cells. GAPDH was used as a loading control. (D) Cell Counting Kit-8 assay was performed to determine the cell viability of A2780-Ctrl, A2780-TIPE1 and A2780-TIPE1 cells treated with the z-VAD inhibitor. (n=5). (E) Determination of apoptotic cells by PI and Annexin V staining in A2780-Ctrl, A2780-TIPE1 and A2780-TIPE1 cells treated with the z-VAD inhibitor, followed by flow cytometry analysis. The percentage of apoptotic cells was analyzed ( $\mathrm{n}=5) .{ }^{* *} \mathrm{P}<0.01 \mathrm{vs}$. Ctrl; ${ }^{\# /} \mathrm{P}<0.01$ vs. TIPE1. TIPE1, tumor necrosis factor- $\alpha$-induced protein 8 -like 1; PI, propidium iodide; CC, cleaved caspase; PARP, cleaved poly(ADP-ribose) polymerase; Ctrl, control; OD, optical density. 
A

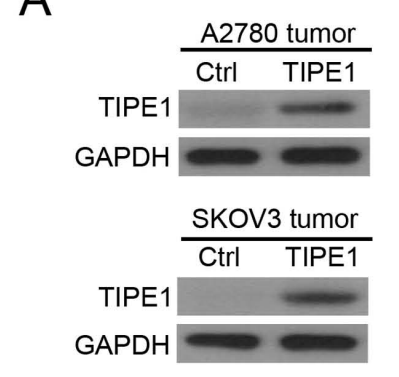

B

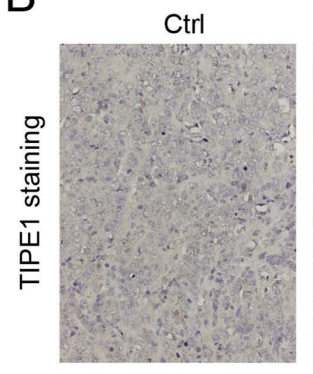

TIPE1

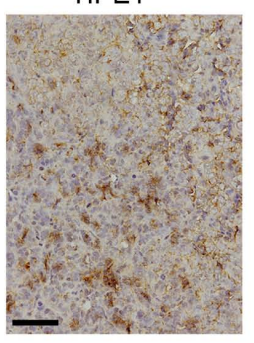

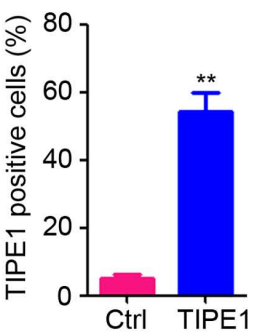

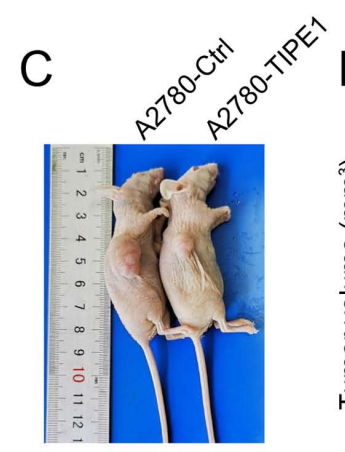

D

A2780

E

A2780
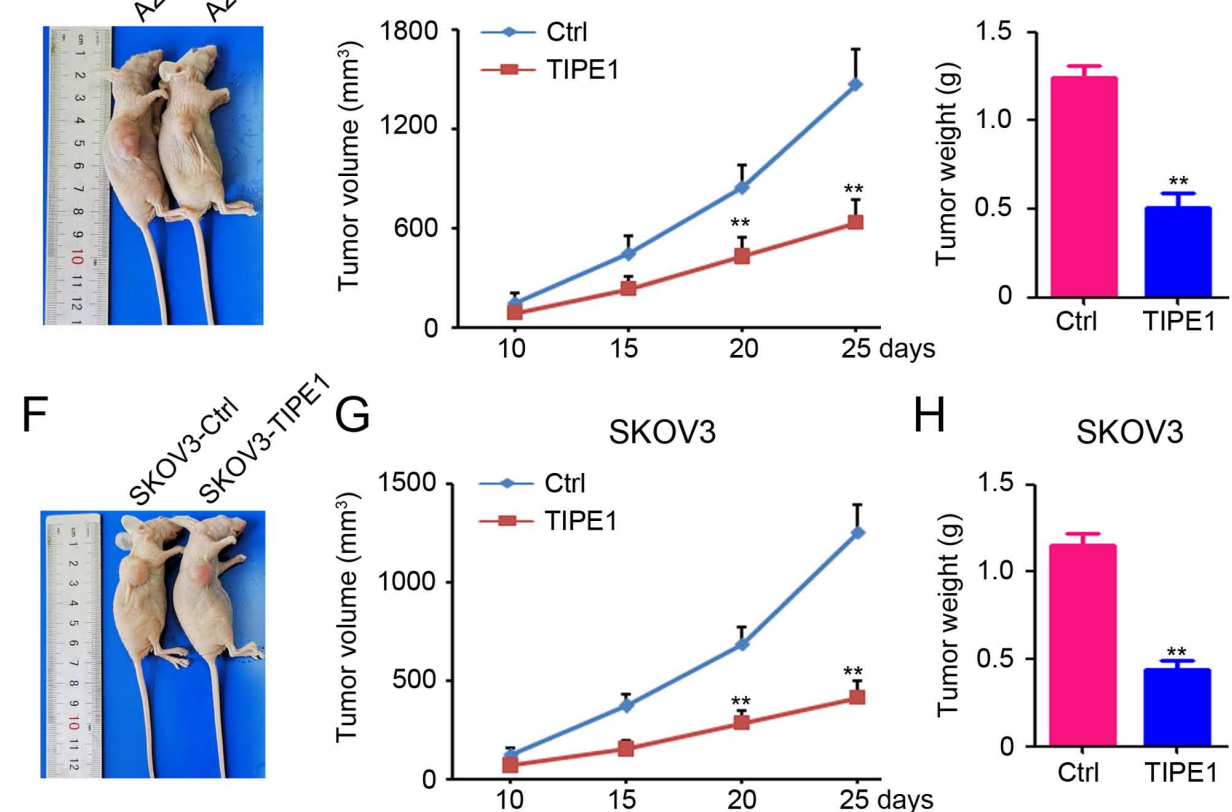

$\mathrm{H}$

SKOV3

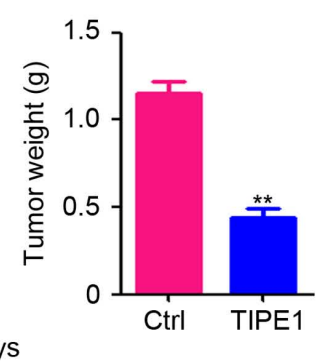

Figure 4. TIEP1 impairs ovarian tumor growth in vivo. (A) Western blot analysis of TIPE1 expression in A2780 and SKOV3 tumors. GAPDH was used a loading control. (B) Immunohistochemical staining of TIPE1 expression in A2780 tumors. The percentage of TIPE1 positive cells were counted and analyzed ( $\mathrm{n}=4$ /group). Scale bar, $100 \mu \mathrm{m}$. (C) Image of mice with A2780-Ctrl or A2780-TIPE1 tumor xenografts. (D) Tumor volume of A2780-Ctrl and A2780-TIPE1 tumors at 10, 15, 20 and 25 days after cell injection (n=6/group). (E) Tumor weight of A2780-Ctrl and A2780-TIPE1 tumors at 25 days after cell injection ( $\mathrm{n}=6 /$ group). (F) Image of mice with SKOV3-Ctrl or SKOV3-TIPE1 tumor xenografts. (G) Tumor volume of SKOV3-Ctrl and SKOV3-TIPE1 tumors at 10, 15, 20 and 25 days after cell injection ( $\mathrm{n}=6$ /group). (H) Tumor weight of SKOV3-Ctrl and SKOV3-TIPE1 tumors at 25 days after cell injection ( $\mathrm{n}=6 / \mathrm{group}$ ). ${ }^{* *} \mathrm{P}<0.01$ vs. Ctrl. TIPE1, tumor necrosis factor- $\alpha$-induced protein 8 -like 1; Ctrl, control.

results suggest that TIPE1 promotes caspase-dependent apoptosis in ovarian cancer.

TIPE1 impairs ovarian tumor growth in vivo. To further confirm the functional role of TIPE1 in ovarian cancer, a xenograft mouse model was established by subcutaneously injecting A2780 or SKOV3 cells into the right ventricles of mice. Western blot analysis demonstrated the upregulation of TIPE1 expression in both A2780-TIPE1 and SKOV3-TIPE1 tumors compared with A2780-Ctrl and SKOV3-Ctrl tumors, respectively (Fig. 4A). In addition, a larger number of TIPE1-positive cells were observed in A2780-TIPE1 tumors compared with A2780-Ctrl tumors (Fig. 4B). As presented in Fig. 4C-E, ectopic TIPE1 expression effectively inhibited A2780 tumor growth, with a 56.9\% reduction in tumor volume (Fig. 4D; A2780-Ctrl tumor volume, $1467.2 \pm 215.4 \mathrm{~mm}^{3}$ vs. A2780-TIPE1 tumor volume, $632.9 \pm 132.8 \mathrm{~mm}^{3}$ ), and a $59.0 \%$ reduction in tumor weight (Fig. $4 \mathrm{E}$; A2780-Ctrl tumor weight, $1.24 \pm 0.16 \mathrm{~g}$ vs. A2780-TIPE1 tumor weight, $0.51 \pm 0.20 \mathrm{~g}$ ). Furthermore, a significant antitumor effect of TIPE1 was also observed in SKOV3 tumors, with a $67.1 \%$ reduction in tumor volume (Fig. 4F and G; SKOV3-Ctrl tumor volume, $1254.3 \pm 139.4 \mathrm{~mm}^{3}$ vs. SKOV3-TIPE1 tumor volume, $412.8 \pm 83.4 \mathrm{~mm}^{3}$ ), and a $61.8 \%$ reduction in tumor weight (Fig. $4 \mathrm{H}$; SKOV3-Ctrl tumor weight, $1.15 \pm 0.17 \mathrm{~g}$ vs. SKOV3-TIPE1 tumor weight, $0.44 \pm 0.13 \mathrm{~g}$ ). Collectively, these results suggest that TIPE1 impairs ovarian tumor growth in vivo.

TIPE1 promotes ovarian tumor cell apoptosis. To determine the underlying molecular mechanism by which TIPE1 inhibits ovarian tumor growth in vivo, TUNEL assay was performed to detect apoptotic cells in A2780 and SKOV3 tumors. As presented in Fig. 5A, a significantly greater number of apoptotic cells were observed in A2780-TIPE1 tumors compared with A2780-Ctrl tumors. A similar result was demonstrated for SKOV3 tumors (Fig. 5B). Furthermore, caspase-3 expression was measured in A2780 and SKOV3 tumors by IHC staining, and the results indicated that the percentage of caspase 3-positive cells was significantly increased in both 
A

A2780 tumor

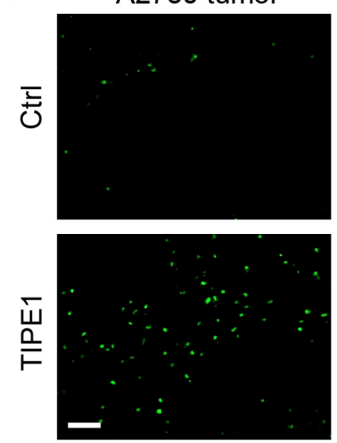

C

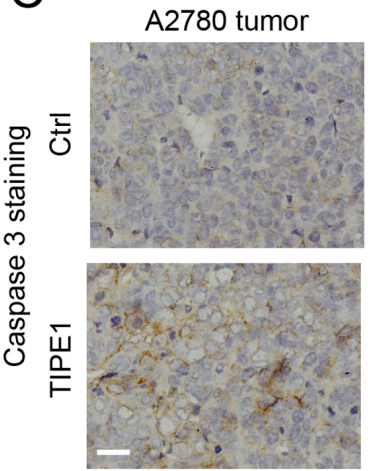

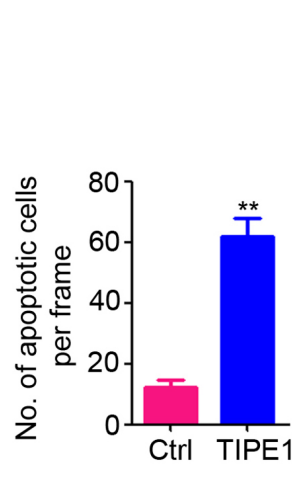

B
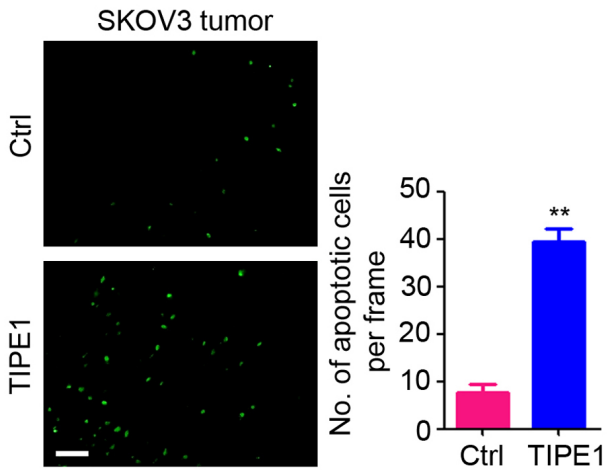
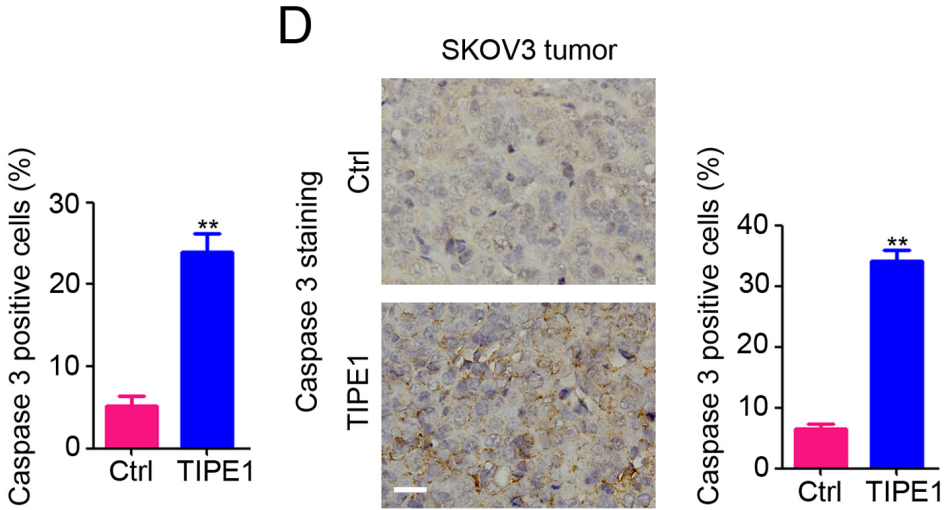

Figure 5. TIPE1 promotes ovarian tumor cell apoptosis. (A and B) Detection of apoptotic cells via the terminal-deoxynucleoitidyl transferase mediated nick end labeling assay in A2780 and SKOV3 tumors. The number of apoptotic cells in each frame was counted and analyzed (n=4/group). Scale bar, $100 \mu \mathrm{m}$. (C and D) Detection of caspase-3 positive cells via immunohistochemical staining in A2780 and SKOV3 tumors. The number of caspase-3 positive cells in each frame was counted and analyzed ( $\mathrm{n}=4$ /group). Scale bar, $100 \mu \mathrm{m} .{ }^{* *} \mathrm{P}<0.01 \mathrm{vs}$. Ctrl. TIPE1, tumor necrosis factor- $\alpha$-induced protein 8 -like 1 ; Ctrl, control.

A2780-TIPE1 (Fig. 5C) and SKOV3-TIPE1 tumors (Fig. 5D). Collectively, these results indicate that TIPE1 induces ovarian tumor cell apoptosis by promoting caspase 3 expression, which was consistent with the in vitro results.

\section{Discussion}

As an important regulator of homeostasis, the TIPE1 protein has been detected in a wide variety of normal tissues, including neurons, hepatocytes, muscular tissues and epithelial cells (23). Furthermore, several studies have demonstrated the dysregulation of TIPE1 in various types of cancer, including lung cancer (14-17,24). The majority of these studies have reported the downregulation of TIPE1 expression in cancer tissues and cells (14-16). However, enhanced TIPE1 expression has been reported in cervical cancer tissues and cells (17). In the present study, TIPE1 expression was demonstrated to be downregulated in malignant tissues of patients with ovarian cancer and ovarian cancer cells, which was consistent with the results of previous studies on lung cancer (15), hepatocellular carcinoma (14) and gastric cancer (16). Based on the results demonstrating altered TIPE1 expression in cancer tissues, the potential role of TIPE1 in predicting patient prognosis was also assessed in the present study. This has also been assessed in other types of cancer in previous studies $(14,15,17)$. The results of the present study confirmed an inverse association between TIPE1 expression and poor prognosis in patients with ovarian cancer, which expands the role of TIPE1 in predicting prognosis in various types of cancer.
TIPE1 plays crucial roles in diverse pathological and physiological processes. In sepsis, TIPE1 functions as a protective factor by inhibiting dendritic cell maturation, and subsequently suppressing T-cell-mediated immunity (25). Antitumor effects of TIPE1 have also been demonstrated in several types of cancer, including colorectal and breast cancer $(24,25)$. Recently, Ye et al (26) reported that ectopic TIPE1 expression efficiently impairs stemness in colorectal cancer both in vitro and in vivo. TIPE1 also inhibits breast cancer cell proliferation, both in vivo and in vitro (27), whereas it serves as an oncogene in the pathogenesis of cervical cancer (17). Furthermore, TIPE1 overexpression effectively inhibits osteosarcoma tumor growth in vivo, which may contribute to the inhibition of macrophage infiltration in osteosarcoma (18). In the present study, the tumor suppressor role of TIPE1 in ovarian cancer was confirmed by promoting apoptosis in vitro and in vivo. Collectively, the results of the present study suggest that TIPE1 may function as a potential therapeutic target for ovarian cancer. Prospective studies will focus on investigating the association and role of TIPE1 in chemotherapy-induced apoptosis in ovarian cancer.

Promoting apoptosis is an efficient strategy for inhibiting tumor progression and promoting chemotherapy sensitivity in different types of cancer, including ovarian cancer $(28,29)$. Curcumin induces apoptosis in a dose- and time-dependent manner by increasing the cytosolic $\mathrm{Ca}^{2+}$ concentration in ovarian cancer cells (30). Luteolin promotes apoptosis in cisplatin-resistant ovarian cancer cells and enhances the cisplatin-induced reduction of tumor growth in 
mice (31). The results of the present study demonstrated the apoptosis-promoting role of TIPE1 in ovarian cancer cells. In addition, ectopic TIPE1 expression was demonstrated to impair ovarian cancer tumor growth in mice by inducing apoptosis. Further investigations of the underlying molecular mechanism indicated that TIPE1 increased the expression of caspase-3, caspase- 8 and caspase- 9 in ovarian cancer cells. Inhibition of caspase activity blocked the induction of apoptosis by TIPE1 in ovarian cancer cells. $\beta$-catenin, Rac1 and p53 acetylation have been reported as the downstream targets of TIPE1 for the regulation of tumor growth $(14,17,26)$. Thus, further studies are required to identify the downstream targets of TIPE1 for the regulation of ovarian cancer cell proliferation.

In conclusion, the results of the present study demonstrated the prognosis-predicting role and antitumor activity of TIPE1 in ovarian cancer. However, further studies are required to identify the direct targets of TIPE1 for the promotion of caspase-dependent apoptosis. Furthermore, there is a long way to demonstrate the therapeutic effect of TIPE1 in patients with ovarian cancer.

\section{Acknowledgements}

Not applicable.

\section{Funding}

The present study was supported by grants from The National Natural Science Foundation of China (grant no. 81871214) and the Merck Serono China Research Fund for Fertility Experts.

\section{Availability of data and materials}

All data generated and/or analyzed during this study are included in this published article.

\section{Authors' contributions}

SQ designed the experimental objective and made the final decision for the manuscript. TL and LJ performed the main experiments, and TL drafted the initial manuscript and performed the literature review. YD helped with animal experiments. BW performed the morphology experiments. All authors read and approved the final manuscript.

\section{Ethics approval and consent to participate}

All animal experiments were approved by the Ethics Committee of Southern Medical University (20180416, Guangzhou, China), and complied with the animal experiment guidelines of Southern Medical University.

\section{Patient consent for publication}

Not applicable.

\section{Competing interests}

The authors declare that they have no competing interests.

\section{References}

1. Torre LA, Trabert B, DeSantis CE, Miller KD, Samimi G, Runowicz CD, Gaudet MM, Jemal A and Siegel RL: Ovarian cancer statistics, 2018. CA Cancer J Clin 68: 284-296, 2018.

2. Siegel RL, Miller KD and Jemal A: Cancer statistics, 2020. CA Cancer J Clin 70: 7-30, 2020.

3. Lheureux S, Braunstein M and Oza AM: Epithelial ovarian cancer: Evolution of management in the era of precision medicine. CA Cancer J Clin 69: 280-304, 2019.

4. Partridge EE and Barnes MN: Epithelial ovarian cancer: Prevention, diagnosis, and treatment. CA Cancer J Clin 49: 297-320, 1999.

5. Kim JY, Cho $\mathrm{CH}$ and Song HS: Targeted therapy of ovarian cancer including immune check point inhibitor. Korean J Intern Med (Korean Assoc Intern Med) 32: 798-804, 2017.

6. Kroeger PT Jr and Drapkin R: Pathogenesis and heterogeneity of ovarian cancer. Curr Opin Obstet Gynecol 29: 26-34, 2017.

7. Dong X, Men X, Zhang W and Lei P: Advances in tumor markers of ovarian cancer for early diagnosis. Indian J Cancer 51 (Suppl 3): e72-e76, 2014.

8. Yang WL, Lu Z and Bast RC Jr: The role of biomarkers in the management of epithelial ovarian cancer. Expert Rev Mol Diagn 17: 577-591, 2017.

9. Bordoloi D, Banik K, Shabnam B, Padmavathi G, Monisha J, Arfuso F, Dharmarajan A, Mao X, Lim LHK, Wang L, et al: TIPE family of proteins and its implications in different chronic diseases. Int J Mol Sci 19: 19, 2018.

10. Jiang J, Pang X, Liu H, Yang X, Zhang Y, Xiang X, Li J, Li T and Zhao P: Reduced TIPE2 expression is inversely associated with proinflammatory cytokines and positively correlated with bone mineral density in patients with osteoporosis. Life Sci 216: 227-232, 2019.

11. Wang Q, Ma L, Liu T, Ge C, Zhou Q, Wei C and Shi W: TIPE2 suppresses Pseudomonas aeruginosa keratitis by inhibiting NF-kappaB signaling and the infiltration of inflammatory cells. J Infect Dis 220: 1008-1018, 2019.

12. Liu Y, Ni XY, Chen RL, Li J and Gao FG: TIPE attenuates the apoptotic effect of radiation and cisplatin and promotes tumor growth via JNK and p38 activation in Raw264.7 and EL4 cells. Oncol Rep 39: 2688-2694, 2018.

13. Padmavathi G, Banik K, Monisha J, Bordoloi D, Shabnam B, Arfuso F, Sethi G, Fan L and Kunnumakkara AB: Novel tumor necrosis factor- $\alpha$ induced protein eight (TNFAIP8/TIPE) family: Functions and downstream targets involved in cancer progression. Cancer Lett 432: 260-271, 2018.

14. Zhang Z, Liang X, Gao L, Ma H, Liu X, Pan Y, Yan W, Shan H, Wang Z, Chen YH, et al: TIPE1 induces apoptosis by negatively regulating Rac1 activation in hepatocellular carcinoma cells. Oncogene 34: 2566-2574, 2015.

15. Wu X, Ma Y, Cheng J, Li X, Zheng H, Jiang L and Zhou R: TIPE1 function as a prognosis predictor and negative regulator of lung cancer. Oncotarget 8: 78496-78506, 2017.

16. Liu W, Chen Y, Xie H, Guo Y, Ren D, Li Y, Jing X, Li D, Wang X, Zhao M, et al: TIPE1 suppresses invasion and migration through down-regulating Wnt/ $\beta$-catenin pathway in gastric cancer. J Cell Mol Med 22: 1103-1117, 2018.

17. Zhao P, Pang X, Jiang J, Wang L, Zhu X, Yin Y, Zhai Q, Xiang X, Feng $\mathrm{F}$ and $\mathrm{Xu}$ W: TIPE1 promotes cervical cancer progression by repression of p53 acetylation and is associated with poor cervical cancer outcome. Carcinogenesis 40: 592-599, 2019.

18. Chen P, Zhou J, Li J, Zhang Q and Zuo Q: TIPE1 suppresses osteosarcoma tumor growth by regulating macrophage infiltration. Clin Transl Oncol 21: 334-341, 2019.

19. Dai L, Cui X, Zhang X, Cheng L, Liu Y, Yang Y, Fan P, Wang Q, Lin Y, Zhang J, et al: SARI inhibits angiogenesis and tumour growth of human colon cancer through directly targeting ceruloplasmin. Nat Commun 7: 11996, 2016.

20. Ujiie D, Okayama H, Saito K, Ashizawa M, Min AK, Endo E, Kase K, Yamada L, Kikuchi T, Hanayama H, et al: KRT17 as a prognostic biomarker for stage II colorectal cancer. Carcinogenesis 41: 591-599, 2020.

21. Bao L, You B, Shi S, Shan Y, Zhang Q, Yue H, Zhang J, Zhang W, Shi Y, Liu Y, et al: Metastasis-associated miR-23a from nasopharyngeal carcinoma-derived exosomes mediates angiogenesis by repressing a novel target gene TSGA10. Oncogene 37: 2873-2889, 2018.

22. Livak KJ and Schmittgen TD: Analysis of relative gene expression data using real-time quantitative PCR and the 2(-Delta Delta C(T)) method. Methods 25: 402-408, 2001. 
23. Cui J, Zhang G, Hao C, Wang Y, Lou Y, Zhang W, Wang J and Liu S: The expression of TIPE1 in murine tissues and human cell lines. Mol Immunol 48: 1548-1555, 2011.

24. Wang Y, Liu Y, Hu C, Ni X and Huang X: Tumor necrosis factor $\alpha$-induced protein 8 -like 1 promotes apoptosis by regulating B-cell leukemia/lymphoma-2 family proteins in RAW264.7 cells. Oncol Lett 12: 3506-3512, 2016.

25. Luan YY, Zhang L, Zhu FJ, Dong N, Lu JY and Yao YM: Effect of TIPE1 on immune function of dendritic cells and its signaling pathway in septic mice. J Infect Dis 220: 699-709, 2019.

26. Ye T, Yang B, Wang C, Su C, Luo J, Yang X, Yu H, Yuan Z, Meng Z and Xia J: TIPE1 impairs stemness maintenance in colorectal cancer through directly targeting $\beta$-catenin. Carcinogenesis 41 : 25-35, 2020

27. Hu W, Feng CM, Liu LY, Li N, Tian F, Du JX, Zhao Y, Xiang XX, Liu $\mathrm{K}$ and Zhao PQ: TIPE1 inhibits breast cancer proliferation by downregulating ERK phosphorylation and predicts a favorable prognosis. Front Oncol 9: 400, 2019.

28. Tong Y, Zhang G, Li Y, Xu J, Yuan J, Zhang B, Hu T and Song G: Corilagin inhibits breast cancer growth via reactive oxygen species-dependent apoptosis and autophagy. J Cell Mol Med 22: $3795-3807,2018$.
29. Yan XY, Zhong XR, Yu SH, Zhang LC, Liu YN, Zhang Y, Sun LK and Su J: p62 aggregates mediated caspase 8 activation is responsible for progression of ovarian cancer. J Cell Mol Med 23. 4030-4042, 2019.

30. Seo JA, Kim B, Dhanasekaran DN, Tsang BK and Song YS: Curcumin induces apoptosis by inhibiting sarco/endoplasmic reticulum $\mathrm{Ca}^{2+}$ ATPase activity in ovarian cancer cells. Cancer Lett 371: 30-37, 2016

31. Wang H, Luo Y, Qiao T, Wu Z and Huang Z: Luteolin sensitizes the antitumor effect of cisplatin in drug-resistant ovarian cancer via induction of apoptosis and inhibition of cell migration and invasion. J Ovarian Res 11: 93, 2018.

(i) () $(9)$ This work is licensed under a Creative Commons Attribution-NonCommercial-NoDerivatives 4.0 International (CC BY-NC-ND 4.0) License. 